\title{
The first BDJ Clinician's Guide is published
}

The British Dental Journal is proud to announce the publication of the first in a new series of books: the BDJ Clinician's Guides.

Published in association with the British Dental Association (BDA), this new series will build on the trusted BDJ Clinical Guides to create books that update and inform the busy clinician on key topics across all fields of dentistry. Each volume is authored by recognised experts, is superbly illustrated and provides concise, highly practical guidance and solutions.

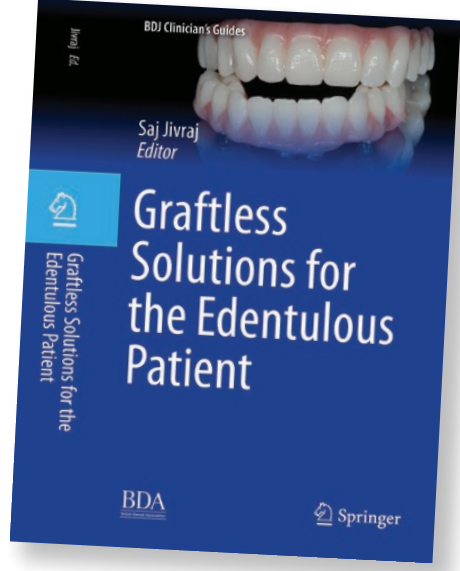

The first book in the series is Graftless Solutions for the Edentulous Patient, edited by Dr Saj Jivraj. This guide explains the rationale and technique for the rehabilitation of fully edentulous patients, covering both traditional graftless concepts and zygomatic implant strategies. It provides step-bystep descriptions of techniques and offers guidance on management of complications.

The book is available to buy now from the BDA shop: www.bda.org/shop.

BDA members receive a $22.5 \%$ discount and free $P \& P$.

\section{Prosthetic Dentistry Department reunion planned}

1986-1988 alumni of the Prosthetic Dentistry Department, Eastman Dental Institute, are invited to attend a reunion event to coincide with the 66th Annual Conference of the British Society of Prosthodontics (BSSPD) in London on 15-16 March 2019, and the shifting of the Eastman Dental Institute (EDI) to its new location close to UCL's main campus.
Prosthetic dentistry students who graduated in 1986 to 1988 are invited to attend the event along with staff including clinical teachers, office and clinic secretaries, nurses/dental surgery assistants, technical and laboratory staff and instructors who worked during the same period in the Prosthetic Dentistry Department at the Eastman.

To facilitate organisation of the event and maximum participation, graduates and staff from 1986-88 are requested to send their details and contact information to: Professor Dr Fazal Ghani, Dean Postgraduate Dental Studies \& Head of Prosthodontics Department, Peshawar Dental College, Warsak Road, Peshawar 25160, Pakistan.

Telephone number: +92 (091) 520 1848; mobile +92 (0301) 594 3425; email fazalg55@hotmail.com.

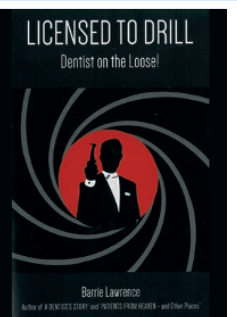

\section{LICENSED TO DRILL}

Barrie Lawrence

Grosvenor House Publishing Limited, 2017

price $\mathrm{f} 8.99 \mathrm{pp} .238$

ISBN: 9781786238412

Although not every dental professional may choose to read dental themed literature for pleasure, some amusement may be found in this text. A light-hearted read, Barrie Lawrence's latest book presents a series of well-intentioned dental anecdotes from a long career in the profession.

As the title suggests, readers can expect tireless James Bond references throughout the book, with chapters such as 'Goldentooth', 'On her Majesty's National Health Service' and 'For Your Teeth Only' setting the tone. The content is a series of pun-heavy stories throughout which the author, with a hint of realism, compares the daily work of a general dentist to the activities of a secret service agent. Progressing through the book, the puns become repetitive and predictable, which unfortunately can detract from the narrative. As a retired dentist, some of the stories reflect a different time in the profession and show how much change has occurred - a heavy hint of nostalgia is present with an air of reflection into a potentially simpler and less-regulated time.

The author is open from the outset regarding his spiritual beliefs and the resultant impact on his writing - the consequence, specified by the author himself, is an inoffensive text, free of any crude or inappropriate content. On this basis, it is a very accessible book for those wanting to hear about the reality of working with the general public in a challenging profession. The target audience, however, is unclear as dentists are well aware of the reality whilst those outside of the profession may not wish to know the events happening behind-the-scenes in such detail, if any.

In summary, though this may not be the author's intention, the book may be suited to a younger individual considering a career in dentistry as the stories provide some basic history of dentistry and a real insight into the characters faced in daily practice with sprinklings of basic dental science education thrown in. The problem, however, is that it is this exact group that may struggle to tolerate the sense of humour of a different generation. If a reader can prepare to mentally filter out excessive 007 references, this book may provide some light-hearted entertainment.

Andrew Geddis-Regan 